\title{
Ultra-long range distributed fibre sensing using virtually transparent propagation
}

\author{
Miguel Gonzalez-Herraez ${ }^{1}$, Sonia Martin-Lopez ${ }^{2}$, Mercedes Alcon-Camas ${ }^{3}$, Pedro Corredera ${ }^{2}$, Luc \\ Thévenaz ${ }^{4}$ and Juan Diego Ania-Castañon ${ }^{2}$ \\ ${ }^{1}$ Departamento de Electronica, Universidad de Alcala, Campus Universitario s/n, 28871, Alcala de Henares, Madrid, Spain \\ ${ }^{2}$ Instituto de Óptica, Consejo Superior de Investigaciones Científicas, C/ Serrano 144, Madrid, 28006, Spain \\ ${ }^{3}$ Photonics Research Group, Aston University, Aston Triangle, Birmingham B4 7ET, UK \\ ${ }^{4}$ Group for Fibre Optics, Ecole Polytechnique Federale de Lausanne, STI IEL GR-SCI Station 11, CH-1015 Lausanne, Switzerland
}

Distributed fibre sensors provide unique capabilities for monitoring large infrastructures with high resolution. Practically, all these sensors are based on some kind of backscattering interaction. A pulsed activating signal is launched on one side of the sensing fibre and the backscattered signal is read as a function of the time of flight of the pulse along the fibre. A key limitation in the measurement range of all these sensors is introduced by fibre attenuation. As the pulse travels along the fibre, the losses in the fibre cause a drop of signal contrast and consequently a growth in the measurement uncertainty. In typical single-mode fibres, attenuation imposes a range limit of less than $30 \mathrm{~km}$, for resolutions in the order of 1-2 meters. An interesting improvement in this performance can be considered by using distributed amplification along the fibre [1]. Distributed amplification allows having a more homogeneous signal power along the sensing fibre, which also enables reducing the signal power at the input and therefore avoiding nonlinearities. However, in long structures (>50 km), plain distributed amplification does not perfectly compensate the losses and significant power variations along the fibre are to be expected, leading to inevitable limitations in the measurements. From this perspective, it is simple to understand intuitively that the best possible solution for distributed sensors would be offered by a virtually transparent fibre, i.e. a fibre exhibiting effectively zero attenuation in the spectral region of the pulse. In addition, it can be shown that lossless transmission is the working point that allows the minimization of the amplified spontaneous emission (ASE) noise build-up.

Virtual transparency in optical fibres can be achieved through the use of higher-order Raman amplification in ultra-long fibre lasers, where the sensing fibre acts also as a cavity for a lasing mode one Raman shift above the signal frequency [2]. In these structures, the gain can be distributed very evenly along the fibre, leading to a close-to-perfect compensation of the losses. Our talk will address theoretically and experimentally the concept of virtual transparency in optical fibres and the means to achieve it. We will show experimental results in which virtual transparency in an ultra-long Raman fibre laser leads to extending the range of a Brillouin Optical TimeDomain Analysis Sensor (BOTDA) setup up to $100 \mathrm{~km}$ keeping a 2 meter measurement resolution. Fig. 1 displays two sample traces obtained with different amplification options in the same sensing fibre $(50 \mathrm{~km})$, one with no amplification and another one using the virtual transparency created by ultra-long laser amplification. As it is visible, the second option guarantees a much better contrast of the measured signal through an extremely homogeneous loss compensation over the whole sensing fibre. As we will show, although ultra-long laser amplification has the advantage of keeping constant contrast across the whole sensing line, there are some issues to address in order for this approach to be the most effective, the most important of which is keeping relative intensity noise transfer from the Raman pumps from affecting the quality of the BOTDA signals.

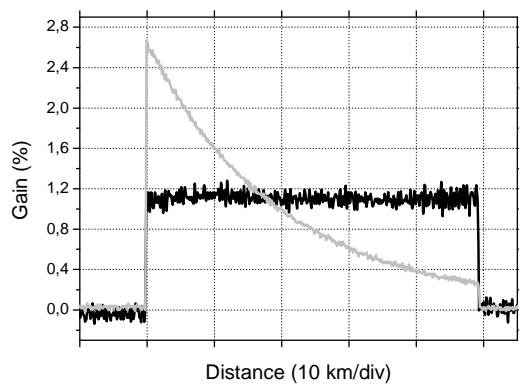

Fig 1. BOTDA traces illustrating the concept of virtual transparency in optical fibres; light gray line, plain BOTDA trace obtained in the sensing fibre with no Raman assistance; black line, using the virtual transparency given by second-order Raman assistance in an ultra-long Raman fibre laser.

\section{References}

[1] F. Rodríguez-Barrios, S. Martín-López, A. Carrasco-Sanz, P. Corredera, J. D. Ania-Castañón, L. Thévenaz, and M. González-Herráez, "Distributed Brillouin Fiber Sensor Assisted by First-Order Raman Amplification," J. Lightwave Technol. 28, 2162-2172 (2010)

[2] S. Martin-Lopez, M. Alcon-Camas, F. Rodriguez, P. Corredera, J. D. Ania-Castañon, L. Thévenaz, and M. Gonzalez-Herraez, "Brillouin optical time-domain analysis assisted by second-order Raman amplification," Opt. Express 18, 18769-18778 (2010) 